\title{
Protective effect of Apelin/APJ system on lipopolysaccharide-related cardiac dysfunction
}

\author{
Junli $\mathrm{Hu}^{1}$, Shuhua Huo ${ }^{1}$, Shiying $\mathrm{Dou}^{2}$, Wei Jiang ${ }^{3}$ and Yongjun $\mathrm{Li}^{4}$ \\ ${ }^{1}$ Emergency Department, The Second Hospital of Hebei Medical University, Shijiazhuang, Hebei, China \\ ${ }^{2}$ Infectious Disease Department, The Second Hospital of Hebei Medical University, Shijiazhuang, Hebei, China \\ ${ }^{3}$ Pathology Department, The Second Hospital of Hebei Medical University, Shijiazhuang, Hebei, China \\ ${ }^{4}$ Cardiovascular Department, The Second Hospital of Hebei Medical University, Shijiazhuang, Hebei, China
}

\begin{abstract}
At present, the pathogenesis of sepsis-induced myocardial dysfunction (SIMD) is not completely clear and effective treatment measures are lacking. Apelin is an endogenous ligand of the angiotensin like G protein coupled receptor APJ and a cardiovascular peptide with multiple functions. Our aim is to analyze the protective effect and mechanism of Apelin/APJ system on lipopolysaccharide (LPS)-induced myocardial dysfunction. One hour before LPS treatment, apelin-13 or an APJ antagonist [Ala]-apelin-13 (F13A) was given for pre-intervention to observe the effect of apelin-13 on cardiac ultrasound, pathological changes and inflammatory factors in LPS-treated mice. Another part of the mice was treated with apelin-13 or apelin-13 combined with F13A one hour after LPS treatment. The results showed that apelin-13 injection significantly reversed the decrease of ejection fraction and the increase of inflammatory factors induced by LPS in mice. Endogenous apelin may have protective effect on SIMD induced by LPS. Exogenous administration of apelin may inhibit LPS-induced inflammation, apoptosis and increase autophagy

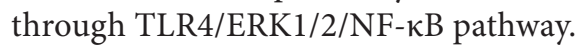

Key words: SIMD — Apelin - APJ — Protective effect

\begin{abstract}
Abbreviations: APJ, angiotensin like G protein coupled receptor; DAPI, 4',6-diamidino-2-phenylindole; EF, ejection fraction; FS, fractional shortening; F13A, [Ala]-apelin-13; LPS, lipopolysaccharide; LVEDV, left ventricular end-diastolic volume; NS, normal saline; SIMD, sepsis-induced myocardial dysfunction; TLR4, toll-like receptor 4 .
\end{abstract}

\section{Introduction}

Sepsis is a common acute and severe disease with a mortality rate of more than 50\% (Jawad et al. 2012), which can cause multiple organ dysfunctions. Cardiovascular system is one of the most important target organs of sepsis (Bentzer et al. 2015). A large number of clinical studies have shown that sepsis-induced myocardial dysfunction (SIMD) and circulatory dysfunction are closely related to the increase of mortality rate in patients with sepsis, and sepsis combined

Correspondence to: Yongjun Li, Cardiovascular Department, The Second Hospital of Hebei Medical University, 215 Heping West Road, Shijiazhuang 050000, Hebei, China

E-mail: lyj13315990301@126.com with cardiac dysfunction is one of the main reasons leading to the increase of mortality (Landesberg et al. 2012; Biering-Sorensen and Solomon 2015). Lipopolysaccharide (LPS) secreted by Gram-negative bacilli is one of the main substances that initiate inflammation and immune imbalance in sepsis (Fry 2012). However, the specific mechanism of SIMD occurrence is still unclear, and there is no effective treatment (Fleischmann et al. 2016). It is considered that the possible mechanisms of SIMD include the inhibitory effect of inflammatory factors on myocardium, apoptosis, mitochondrial dysfunction, calcium influx disorder, etc. (Flierl et al. 2008; Balija and Lowry 2011), and uncontrolled inflammatory response (Takeuchi and Akira 2010) is one of the most important mechanisms. Some scholars believe that similar to myocardial ischemia, myocardial hibernation 
mechanism also exists in sepsis (Levy et al. 2005). Cardiac dysfunction may be the body's self-protection, suggesting that there is an endogenous protection mechanism in the body.

Apelin, an endogenous ligand of APJ, an angiotensin like $\mathrm{G}$ protein coupled receptor, is a polypeptide composed of 77 amino acids (Tatemoto et al. 1998). Recent studies have shown that apelin plays an important role in regulating myocardial contraction, vascular tension, inflammation, immunity, energy metabolism, water balance etc. (Kleinz and Davenport 2005; Galanth et al. 2012; Coquerel et al. 2017). Apelin-13 is one of the important subtypes of apelin, which has strong biological activity, especially in the regulation of cardiovascular function (Szokodi et al. 2002; Charo et al. 2009; Chagnon et al. 2017). Some scholars believed that apelin can enhance myocardial contractility and reduce the load before and after the heart (Ashley et al. 2005). Resent study showed that the activation of APJ by apelin-13 attenuates endoplasmic reticulum stress-related inflammation and oxidative stress after subarachnoid hemorrhage (Xu et al. 2019). However, it is not clear whether the endogenous Apelin/APJ system has a protective effect on LPS-induced cardiac dysfunction.

The aim of this study was to investigate the protective effect of endogenous Apelin/APJ signaling system on cardiac dysfunction induced by LPS in sepsis mice by pretreatment with apelin-13 or [Ala]-apelin-13 (F13A) before LPS injection. In addition, apelin-13 or apelin-13 combined with F13A was given repeatedly after LPS injection to explore the protective effect of apelin on cardiac dysfunction in sepsis mice and its molecular mechanism.

\section{Materials and Methods}

\section{Animals}

Clean healthy male C57BL/6 mice, 6-8 weeks, 18-23 g (raised by Beijing Weitonglihua Laboratory Animal Co., Ltd.), were reared adaptively for one week before the experiment, fed with standard feed and free water intake.

\section{Reagents}

Lipopolysaccharide (LPS) (Sigma Company, USA), apelin-13 (QRPRLS HKGPMPF, Abcam Company, UK), F13A (QRPRLS HKGPMPA, biobyt Company), anti-apelin antibody, anti-ERK (phospho) phosphorylated antibody, anti-NF- $\kappa$ B P65 (phospho) phosphorylated antibody (all purchased from Abcam Company, UK), ERK1/2 antibody, Caspase- 3 antibody, Caspase- 8 antibody, NF- $\kappa$ B P65 antibody, LC3 antibody, TLR4 antibody (all purchased from
Proteintech Company, China), TNF- $\alpha$ and IL-6, apelin-13 ELISA kit (all purchased from Proteintech Company, China), RNA stationary solution RNA later (Thermo Company, USA), TUNEL kit (Roche Company, USA), Vevo 2100 Small Animal Ultrasound Imaging System (Vissonics, Canada).

\section{Experimental grouping}

To prepare sepsis-induced cardiac dysfunction model, C57BL/6 mice were divided into two groups with 4 mice in each group. Healthy control group (control group) ( $n=$ 4): mice were intraperitoneally (i.p.) injected with normal saline (NS). Lipopolysaccharide group (LPS group) $(n=4)$ : mice were i.p. injected with LPS $(20 \mathrm{mg} / \mathrm{kg})$, the modeling time was 24 hours. Cardiac echocardiography, myocardial pathology and ultrastructure were observed. The mRNA and protein levels of apelin in tissue homogenate, tumor necrosis factor $\alpha$ (TNF- $\alpha$ ) and interleukin 6 (IL-6) levels in plasma and myocardial tissue were also observed. The plasma and tissue samples were frozen at $-80^{\circ} \mathrm{C}$ for unified detection.

To observe the protective effect of Apelin/APJ system on LPS-induced cardiac dysfunction and its mechanism, C57BL/6 mice were divided into 7 groups. Healthy control group (control group) $(n=4)$ : i.p. injection of NS. LPS group $(n=4)$ : i.p. injection of LPS $(20 \mathrm{mg} / \mathrm{kg})$, the modeling time was $24 \mathrm{~h}$. Apelin pre-intervention group (apelin+LPS group) $(n=4)$ : i.p. injection of apelin-13 $(1 \mathrm{mg} / \mathrm{kg})$ before i.p. injection of LPS $(20 \mathrm{mg} / \mathrm{kg})$. F13A pre-intervention group (F13A+LPS group) $(n=4)$ : i.p. injection of F13A before i.p. injection of LPS (20 mg/kg). Apelin-13 blank control group (apelin+NS group) $(n=4)$ : mice were pretreated by i.p. injection of apelin-13 $(1 \mathrm{mg} / \mathrm{kg})$, and then given i.p. injection of NS equal to LPS one hour later. Apelin treatment group (LPS+apelin group) $(n=4): 500 \mu \mathrm{g} /$ $\mathrm{kg}$ (Suffredini et al. 1989) of apelin-13 was injected i.p. at one hour after LPS, once every $5 \mathrm{~h}, 4$ times in total. In the apelin + F13 A treatment group (LPS+apelin+F13A group) $(n=4)$, one hour after LPS, apelin-13 combined with F13A was given, the dosage was $500 \mu \mathrm{g} / \mathrm{kg}$, once every $5 \mathrm{~h}$, four times in total. Cardiac echocardiography was performed $24 \mathrm{~h}$ after the LPS or NS injection, followed by heart, lung and blood samples. The pathological changes of myocardium and apoptosis of TUNEL stained cells were observed. The levels of inflammatory factors (TNF- $\alpha$, IL-6), myocardial apoptosis (Caspase-3, Caspase-8) and autophagy (LC3, beclin1) were detected. The contents of myocardial ERK, TLR4 and NF- $\kappa B$ were also measured.

After i.p. injection of LPS for $0.5-1 \mathrm{~h}$, the activity of mice was gradually weakened, with less foraging water, easy to catch, disordered and lusterless fur, less urine, sunken eyeballs, cool body surface, etc. Some mice gradually increased their activity and foraging water within $10 \mathrm{~h}$. 
The mice were anesthetized by intraperitoneal injection of $10 \%$ chloral hydrate $4 \mathrm{ml} / \mathrm{kg}$. The success of anesthesia in mice was indicated by the change of irregular breathing to regular and smooth breathing, relaxation of muscles in the whole body and delayed corneal emission. No animal exhibited signs of peritonitis. All mice survived $24 \mathrm{~h}$ after LPS i.p. injection. The experimental specimens were taken after anesthetizing mice. Carbon dioxide asphyxia (20\% filling rate) was used to euphemize mice, and then neck dislocation occurred. After confirming the death of mice, the corpse was sealed in plastic bags and stored in the animal centers. The animal center entrusts professional organizations to conduct harmless treatment in a unified way. The study was approved by the ethics committee of Second Hospital of Hebei Medical University (approve number: 20116263).

\section{Cardiac echocardiography}

Transthoracic echocardiography was performed $24 \mathrm{~h}$ after LPS administration with high resolution in vivo imaging system of VISUSONICS VEVO2100. The mice were lightly anesthetized with a mixture of $1-2 \%$ isoflurane and oxygen through the nasal cone with an initial inhalation concentration of $2.5 \%$. The mice were fixed on the supine position of the board. The cardiac function parameters of M-shaped images were measured from the short axis images of parasternum in the horizontal direction of papillary muscles. The left ventricular diameters were measured at the end-diastolic and end-systolic stages. The ejection fraction (EF) and fractional shortering (FS) were calculated to evaluate cardiac function. All measurements were performed by a researcher blinded to the treatment, and the data came from at least three cardiac cycles on average.

\section{Histopathological staining}

Myocardial tissue samples were fixed with 4\% paraformaldehyde solution before paraffin embedding. $4-\mu \mathrm{m}$ thickness sections were stained with hematoxylin and eosin (H\&E) or terminal deoxynucleotidyl transferase (TdT) dUTP nick-end labeling (TUNEL) at room temperature. The nucleus was staining by 4',6-diamidino-2-phenylindole (DAPI). The TUNEL positive cells were labeled with green fluorescence and DAPI positive cells were displayed with blue fluorescence. Apoptotic index was calculated as the ratio of TUNEL positive cells to DAPI positive cells.

\section{Transmission electron microscopy}

The specimens were fixed with $4 \%$ glutaraldehyde and stored at $4^{\circ} \mathrm{C}$ until embedding. The tissue samples were then post-fixed in $1 \%$ phosphate-buffered osmium tetroxide and embedded in Eponate 12 resin. The sections were stained with $2 \%$ uranyl acetate and lead citrate at $50 \sim 70 \mathrm{~nm}$. The images were examined by Hitachi H-7500 transmission electron microscope.

\section{ELISA}

The proinflammatory factors, IL- 6 and TNF- $\alpha$, in plasma and myocardial tissue were measured with commercially available ELISA kits. All procedures were performed according to the manufacturer's instructions.

\section{Quantitative real-time fluorescence PCR}

Total myocardial RNA was prepared by in situ lysis with Trizol reagent. The RNA concentration was determined by using the NanoDrop 2000c UV-Vis spectrophotometer (Thermos Scientific, USA). In brief, $0.5 \mu \mathrm{g}$ of total RNA were denatured in the presence of $0.5 \mu$ l oligo dT primer and Random hexamer. In the following experiments, the cDNA was reverse transcribed in a $4 \mu$ lof $5 \times$ iScript Reaction Mix (Bio$\mathrm{Rad})$ at $42^{\circ} \mathrm{C}$ for $30 \mathrm{~min}$. SYBR Green mother liquor was used to complete real-time fluorescence quantitative PCR on step1 plus system (ABI). The amplification curve was used for calculation of Cycle number $(\mathrm{Ct})$ of each reaction. The relative gene expression was calculated through $2^{-\Delta \Delta C t}$ formula. The primers of proteins, abbreviations and accession numbers are as follows: $\beta$-actin (NM_007393.3,287bp): 5'-GTGACGTTGACATCCGTAAAGA-3', 5'-GTAA-

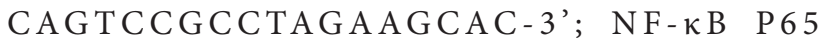
(NM_009045.4,324bp): 5'-AAGCACAGATACCACCAAGACAC-3', 5'-CGCACTGCATTCAAGTCATAGTC-3'; Apelin (NM_013912.4,103bp): 5'-AGTTTGTGGAGTGCCACTGATGT-3',5'-TCCTGGTCCAGTCCTCGAAGTT-3'; Caspase-3 (NM_009810.3,297bp): 5'-TGGAATGTCATCTCGCTCTGGT-3', 5' - AAGAGT T TCG GCTTTCCAGTCA-3'; TLR4 (NM_021297.2,223bp): 5'-TGAGGACTGGGTGAGAAATGAGC-3', 5'-CTGCCATGTTTGAGCAATCTCAT-3'.

\section{Western blot analyses}

Western blot analyses for inflammation/apoptosis/autophage markers and signaling kinases of myocardial tissues were performed. Briefly, the proteins in myocardial tissue lysates were separated by electrophoresis and transferred to a polyvinylidene difluoride membrane. The membranes were blocked and then incubated with antibodies against phosphorylated ERK (p-ERK), ERK1+ERK2, phosphorylated NF- $\kappa \mathrm{B}(\mathrm{p}-\mathrm{NF}-\kappa \mathrm{B}), \mathrm{NF}-\kappa \mathrm{B}$, apelin, active-Caspase-3, Caspase-8, LC3, TLR4, beclin1, in primary antibody working fluid overnight at $4^{\circ} \mathrm{C}$. And then, the membranes were 
incubated with secondary antibody linked to horseradish peroxidase. The band densities were normalized to the density of $\beta$-actin and quantified with scanning densitometric analysis using ImageJ software (National Institutes of Health, Bethesda, Md, USA).

\section{Statistical method}

Statistical evaluation was conducted using SPSS software (18 versions, IBM, Armonk, NY, USA) or GRAPHPAD software (6.02 version, San Diego, CA 92108 USA). One-way ANOVA was used, and then Tukey test (games-howell test for those with uneven variance) was used to test the difference between the treatment groups. The numerical value is expressed as mean value (+SEM). Statistical significance criterion was set as $p<0.05$.

\section{Results}

\section{Cardiac ultrasound results}

The SIMD model was successfully established by intraperitoneal injection of LPS. The expression of apelin in myocardium was decreased by i.p. injection of LPS. EF, FS, left ventricular end-diastolic volume (LVEDV) and stroke volume in LPS group were significantly lower than those in normal control group, with statistical difference $(p<$ 0.05) (Fig. 1A-D). i.p. injection of LPS showed significant cardiac dysfunction in mice. EF, FS, stroke volume and
LVEDV were significantly reduced. Apelin-13 has a protective effect on SIMD induced by LPS, and LPS+apelin group significantly reduced the symptoms of LPS-induced sepsis $(p<0.05)$ (Fig. 1A-D). Compared with LPS group, apelin+LPS and LPS+apelin group significantly improved cardiac function index (Fig. 1A) induced by LPS $(p<0.05)$, respectively.

\section{$\mathrm{H} \& \mathrm{E}$ staining results and ultrastructure}

Compared with the normal control group (Fig. 2A1), H\&E staining showed that the pathological changes of the LPS group were lighter, with only capillary dilation (arrowhead in the Fig. 2A2), granulocyte boundary (curved arrow in the Fig. 2A2), intercellular red blood cell exudation (arrow in the Fig. 2A2), no focal necrosis, inflammatory cell infiltration and interstitial edema (Fig. 2A). Under transmission electron microscope, LPS group has mild swelling of cytoplasm, some mitochondria were slightly or moderate swelling, and fusion of mitochondrial crista and membranes (arrow in the Fig. 2B2), swelling of the endothelial cells of capillaries, sarcomere arranged orderly, and autophagy miniaturous (arrowheads in the Fig. 2B2) were visible in LPS group.

Compared with the LPS group, the pathological changes of myocardial capillary dilation, granulocytic side set and intercellular red blood cell exudation in apelin+LPS group (Fig. 2A3) and LPS+apelin group (Fig. 2A4) were reduced, and myocardial edema and blurred cell border (pentagram in the Fig. 2A4) appeared in the LPS+apelin+F13A group.
A

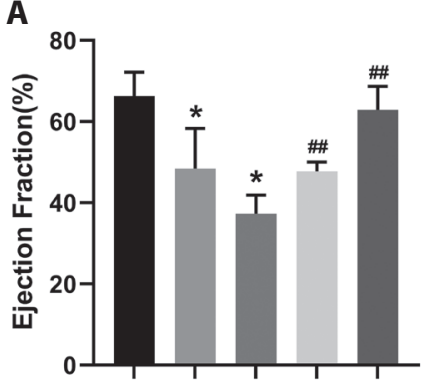

C

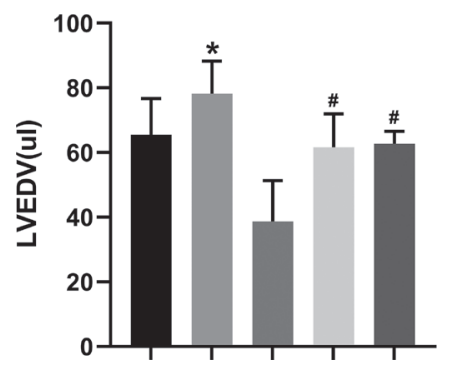

.

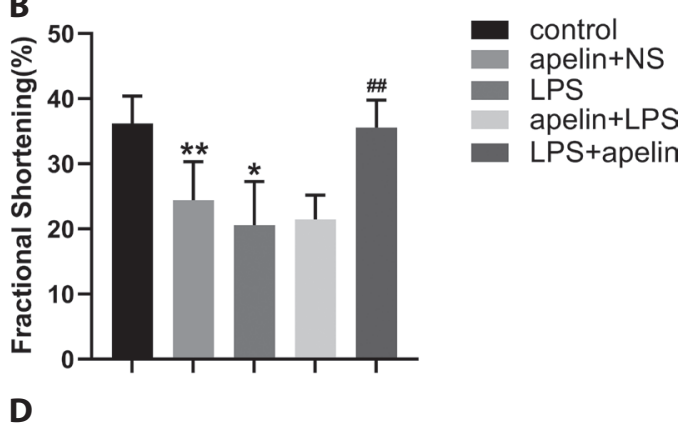

Figure 1. Cardiac echocardiography. Effects of lipopolysaccharide (LPS) injection and apelin-13 on heart rate and cardiac function in mice. apelin $+\mathrm{NS}$, apelin-13 blank control group; apelin+LPS, apelin pre-intervention group; LPS+apelin, apelin treatment group (for detailed description of the groups, see Materials and Methods); LVEDV, left ventricular end-diastolic volume. ${ }^{*} p<0.05,{ }^{* *} p<0.01 v s$. control group; ${ }^{\#} p<0.05,{ }^{\# \#} p<0.01$ vs. LPS group. 

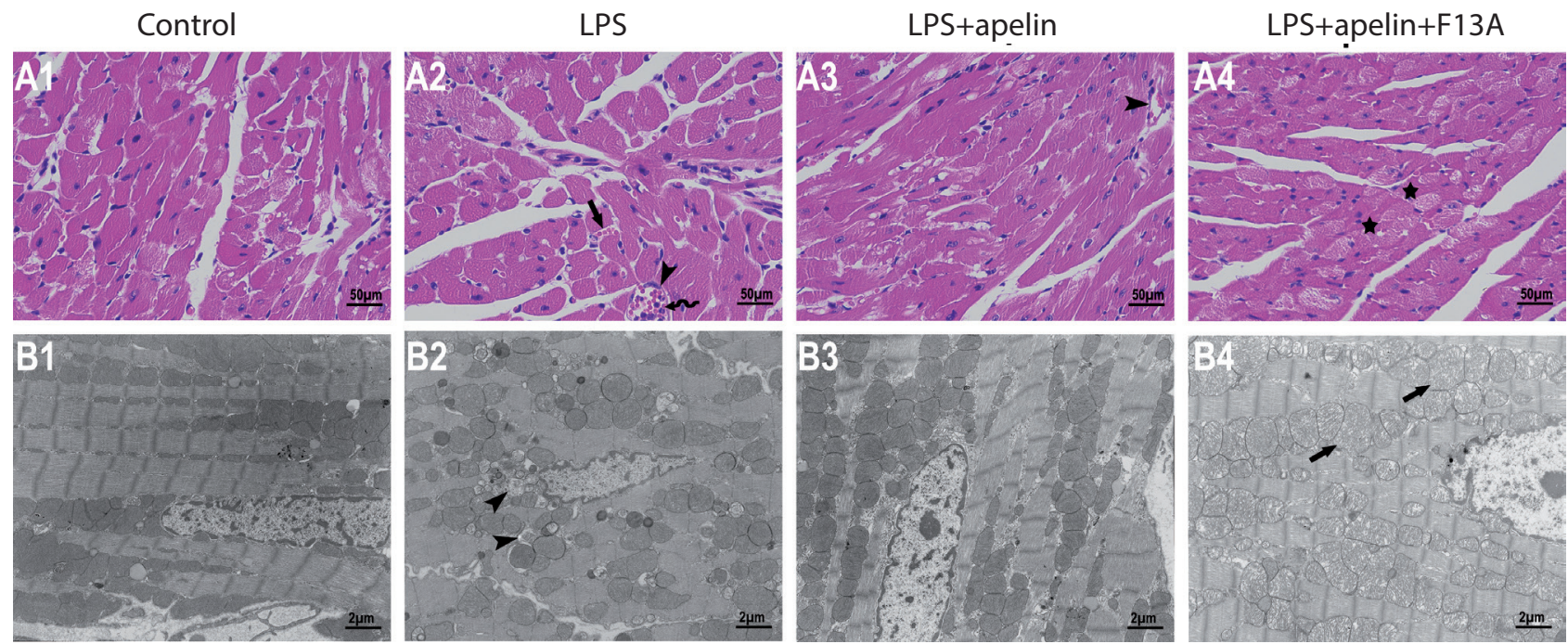

Figure 2. Effects of LPS and apelin-13 on myocardial pathology and ultrastructure. A. H\&E staining was used to observe the pathological changes of normal control group (A1), LPS group (A2), LPS+apelin group (A3) and LPS+apelin+F13A group (A4). Arrows, intercellular red blood cell exudation; arrowheads, capillary dilation; curved arrow, granulocytic side set; asterisks, mild myocardial edema. B. Ultrastructural changes of electron microscopy in each group (B1-B4). Arrows, swelling and fusion of mitochondrial crista and membranes; arrowheads, autophagosome.

\section{Apelin expression level}

LPS intervention significantly reduced the expression of apelin in the myocardium of mice. Western blot (Fig. 3A1) also showed that the level of apelin protein was significantly decreased in LPS group $(p<0.05)$ (Fig. 3A2). The results of real-time fluorescence PCR showed that the expression of apelin in LPS group was significantly lower than that in normal control group, with statistical difference $(p<0.001)$ (Fig. 3A3).

\section{Changes of inflammatory factors in serum and myocardium}

The results of ELISA showed that the levels of IL-6 and TNF- $\alpha$ in serum and myocardium tissues of mice were significantly increased after LPS injection $(p<0.01)$ (Fig. $3 B 1-C 2)$. The serum IL- 6 of apelin+LPS group was lower than that of LPS group $(p<0.001)$ (Fig. 3B1), but there was no statistical significance in the decrease of serum TNF- $\alpha$ (Fig. 3B2) and myocardial tissue IL-6 (Fig. 3C1) and TNF- $\alpha$ $(p>0.05)$ (Fig. 3C2). F13A+LPS group did not aggravate the change of inflammatory factor caused by LPS $(p>0.05)$ (Fig. 3B1-C2). LPS+apelin group significantly inhibited the increase of IL-6 in serum and myocardial tissue induced by LPS ( $p<0.05$ ) (Fig. 3B1, 3C1), but had no significant effect on TNF- $\alpha$ level $(p>0.05)$ (Fig. 3B2,C2). The content of inflammatory factors in LPS+apelin+F13A group was higher than that in LPS+apelin group, but there was no statistical significance $(p>0.05)$ (Fig. 3B1-C2).
Changes of apoptosis and autophagy in cardiomyocytes

TUNEL staining showed that the LPS group had an increase in apoptotic cells compared with the control group, apelin intervention reduced the apoptosis rate, while F13A treatment was given, the number of apoptosis cells increase again (Fig. 4A-E). Western blot results showed that the expression of Cleaved-caspase- 3 and Cleaved-caspase- 8 protein in LPS group was higher than that in control group (Fig. $4 \mathrm{~F}-\mathrm{I})(p<$ 0.001 ), and the expression level of both was significantly reduced in apelin+LPS or LPS+apelin group (Fig. $4 \mathrm{~F}-\mathrm{I})(p<$ 0.001 ), while F13A significantly antagonized the effect of apelin-13 (Fig. 4F-I) $(p<0.001)$. Western blot showed an increase in expression of the beclin 1 proteins in the LPS group compared to the control group $(p<0.001)$, and an increase in expression of both LC3 II (Fig. 4J) and beclin1 proteins (Fig. $4 \mathrm{~K})(p<0.01)$ after the apelin-13 intervention, while F13A significantly inhibited this trend $(p<0.001)$ (Fig. 4J,K).

\section{Effect of apelin-13 on TLR4/ERK/NF- $\kappa B$ signaling pathway}

The Western blot test (Fig. 5) results showed that the apelin intervention significantly inhibited the increase in the expression of myocardial tissue TLR4 protein caused by LPS injection $(p<0.001)$ (Fig. 6A) and the increase in phosphorylation levels of nuclear factor $\mathrm{kBp} 65$ (NF-kBp65) $(p<0.05)$ (Fig. 6B-C), ERK $(p<0.001)$ (Fig. 4D-E), while the effect of F13A was significantly antagonised to apelin-13 $(p<0.05)$. PCR detection showed that TLR4 mRNA level 
A1

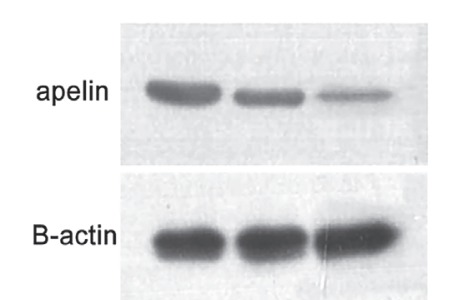

B1

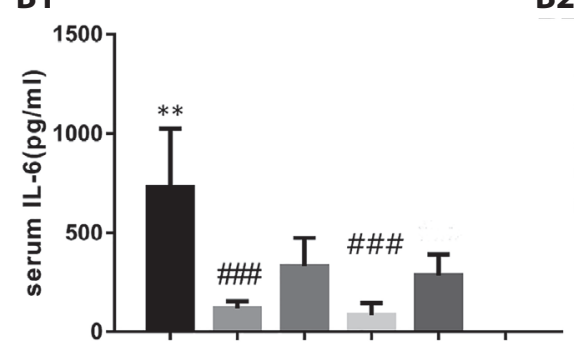

C1

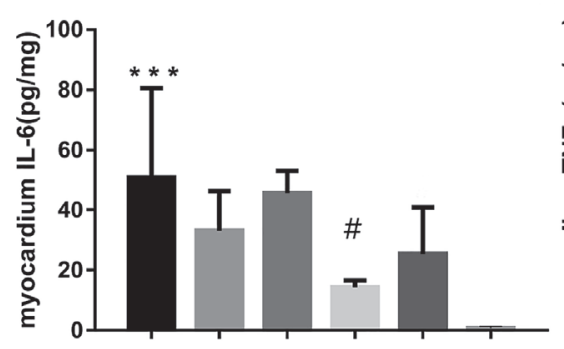

A2

B2
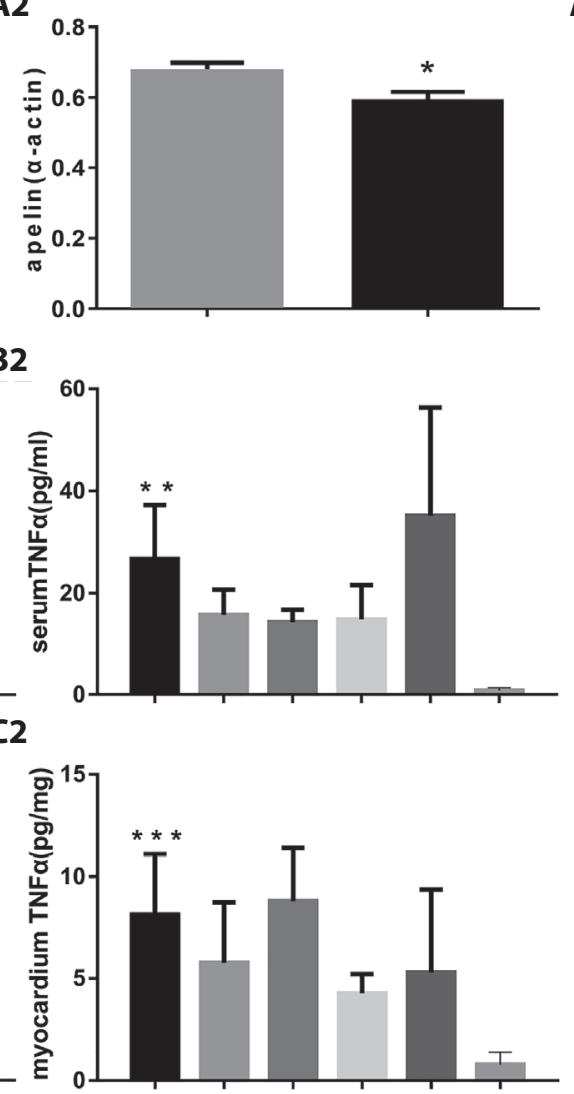

A3

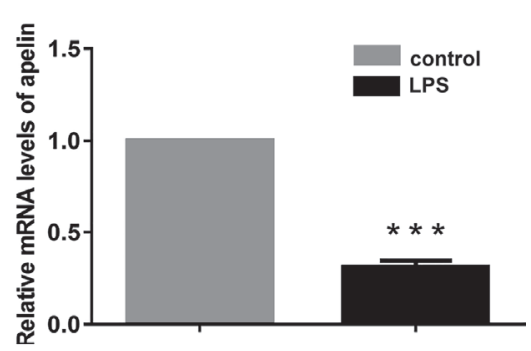

Figure 3. The effect of lipopolysaccharide (LPS) injection on the expression of apelin protein and its mRNA in myocardium (A1-A3). The effects of lipopolysaccharide (LPS) injection and the effects of apelin-13 and F13A intervention on levels of IL- 6 and TNF- $\alpha$ in serum (B1, B2) and myocardium $(\mathbf{C 1}, \mathbf{C 2})$ tissues of mice, detected by ELISA. ${ }^{*} p<0.05$, ${ }^{* *} p<0.01$, ${ }^{* * *} p<0.001 v s$. control group; ${ }^{\#} p<0.05,{ }^{* \#} p<0.01,{ }^{\# \#} p<0.001 v s$. LPS group.

and protein expression trend were the same in each group $(p<0.05)$ (Fig. 6F).

\section{Discussion}

\section{Functional and structural changes of SIMD induced by LPS}

At present, it has been concluded that the cardiac dysfunction (SIMD) caused by sepsis is mainly manifested in the decrease of systolic function and ejection fraction, which is reversible in the surviving patients (Piper et al. 1997). In this study, it was found that LPS could cause cardiac dysfunction in sepsis mice. In addition to the decrease of EF and stroke volume, the decrease of LVEDV was also observed in LPS-induced mice. The latter was different from the increase of LVEDV in clinical study (Farkasfalvi et al. 2007), and the specific mechanism needs further study.

The results of H\&E staining showed that the intercellular red blood cells exuded, the intercellular capillary expanded and the granulocytes gathered, indicating that the permeability of cell membrane increased, but there were no pathological changes such as myocardial edema, focal necrosis and myolysis. This study showed that the significant decrease of heart blood fraction caused by LPS is not caused by severe pathological changes such as myocardial necrosis, which is similar to the conclusion drawn by Piper et al. (1997) using CLP sepsis rat model (Jardin et al. 1999). This result can better explain the reversibility of SIMD.

\section{Effect of apelin-13 on LPS-induced SIMD}

Charo et al. (2009) found that endogenous apelin/APJ had an important regulatory effect on the cardiovascular system, especially in stress situations. Our study found that the mRNA level and protein expression of apelin decreased significantly in the LPS group, suggesting that endogenous apelin may be involved in the development of sepsis.

Apelin is currently considered to be one of the strongest endogenous strong heart substances (Szokodi et al. 2002). In vitro experiments, apelin-13 can significantly enhance the contractility of cardiomyocytes (Farkasfalvi et al. 2007) and isolated myocardium (Szokodi et al. 2002), and it is dose-dependent (Huang et al. 2016). In our study, we found that the increase of EF and heart beat volume, as well as the significant increase of LVEDV were found before or after LPS modeling. Previous clinical studies have shown 

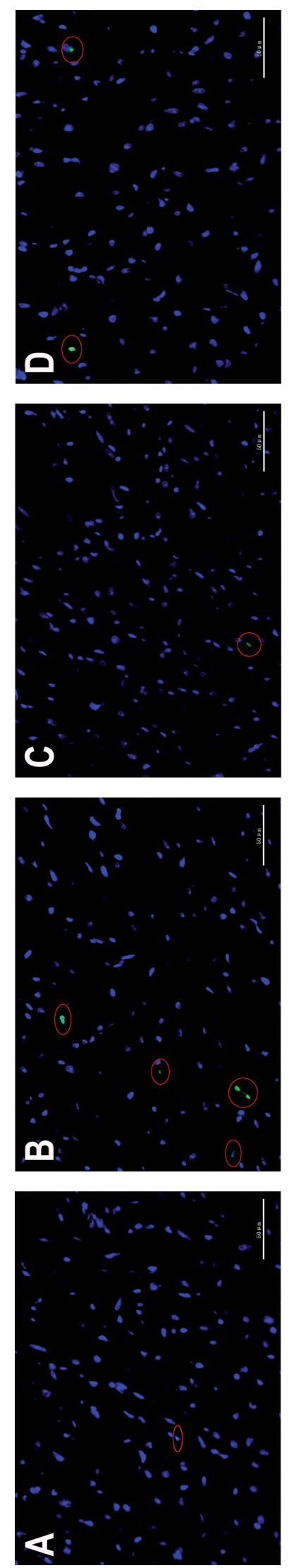
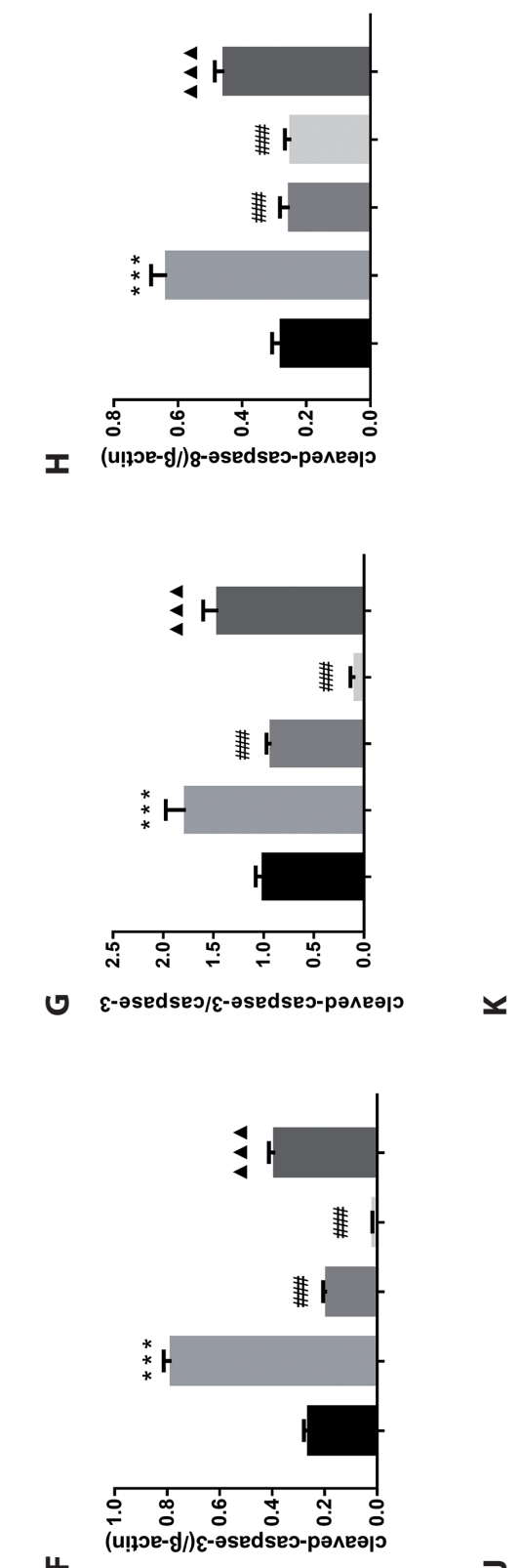

เ

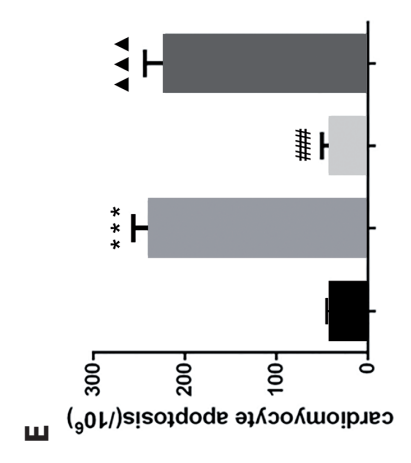

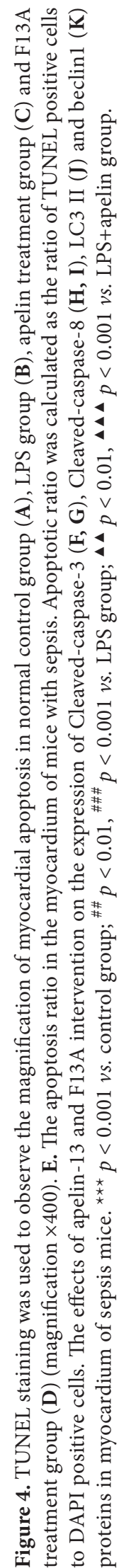


that the relatively low level of LVEDV in sepsis patients is associated with poor prognosis (Jardin et al. 1999). In this study, apelin-13 temporarily increased LVEDV to increase palpitation, improve the tolerance of the heart to fluid, and increase the blood perfusion of organs, thus improving the performance and prognosis of sepsis.

We found that after LPS intervention, apelin-13 significantly reduced IL- 6 in serum and myocardium, while TNF- $\alpha$ was not significantly decreased. The reason may be related to the production and metabolic velocity of the two in the body. TNF- $\alpha$ increased sharply after the injection of LPS, followed by the increase of IL-6, while TNF- $\alpha$ decreased rapidly in vivo (Michie et al. 1988; Fong and Lowry 1990). However, the level of IL-6 in plasma is considered to be more valuable for the prediction of mortality (Sullivan et al. 1992) and nosocomial infection (Dillenseger et al. 2018). Kumar et al. (1999) found that TNF- $\alpha$, IL-6 and other inflammatory factors had inhibitory effect on the contraction of cardiomyocytes, which was positively correlated with the dose. Our results suggested that apelin-13 can inhibit the release of inflammatory factors induced by LPS, and may protect SIMD by reducing the level of inflammatory factors.

\section{L A-L L-A L-F}

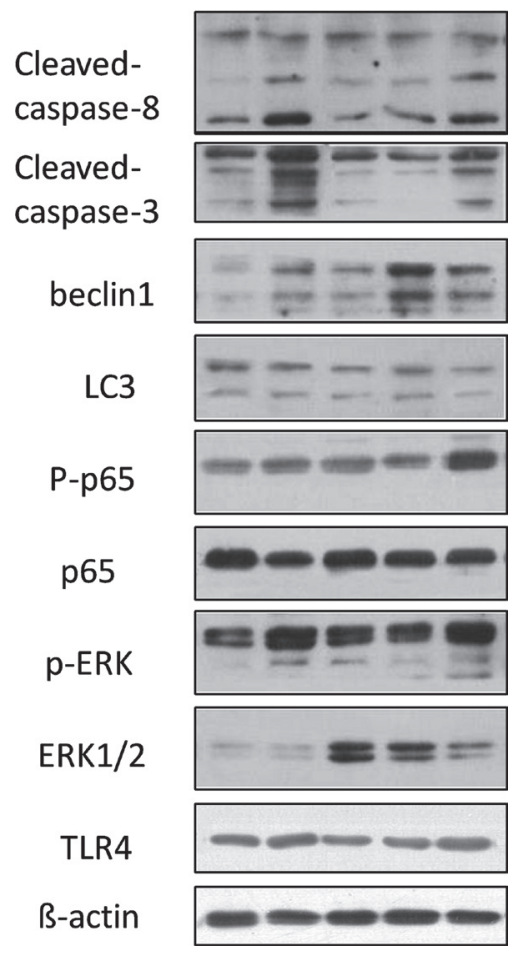

Figure 5. The Western blot result of the Cleaved-caspase-3, Cleaved-caspase-8, LC3, P-p65, p65, ERK1/2, beclin1, TLR4 and p-ERK in myocardium of sepsis mice. C, control group; $\mathrm{L}$, LPS group; A-L, apelin+LPS group; L-A, LPS+apelin group; L-F, LPS+apelin+F13A group.
$\mathrm{NF}-\kappa \mathrm{B}$ is currently considered to play a key role in regulating the release of a variety of inflammatory factors and the complex regulatory network among inflammatory response, immunity and apoptosis (Oeckinghaus et al. 2011; Gasparini and Feldmann 2012; Yang 2017). In the further study of the molecular mechanisms associated with the action of apelin-13, we found that LPS significantly enhanced the phosphorylation level of NF- $\mathrm{kBp} 65$, while the apelin-13 treatment reduced the activity of NF- $\kappa$ B. It was also found that LPS increased the levels of Caspase- 8 and Caspase- 3 in myocardium, while apelin-13 treatment reduced the level of apoptosis. LPS may cause the increase of inflammatory cytokines and apoptosis by activating NF- $\kappa \mathrm{B}$, thus leading to myocardial dysfunction.

At present, it is not entirely clear how the LPS acts on NF- $\kappa$ B. We observed that LPS significantly increased ERK1/2 phosphorylation and TLR4 protein expression, while apelin-13 inhibited this effect. A series of toll-like receptors are expressed in cardiomyocytes (Vaez et al. 2016), such as TLR2, TLR3, TLR4, TLR7, etc. Many studies have confirmed that TLR4 is related to LPS-induced inflammatory response and myocardial injury (Baumgarten et al. 2006; Li et al. 2017; Zhang et al. 2017). LPS produces TNF- $\alpha$ and other inflammatory cytokines, which are activated by toll-like receptors on the surface of cardiomyocytes through NF- $\kappa$ B signaling pathway. These inflammatory cytokines can further activate NF- $\kappa \mathrm{B}$, resulting in cascade reaction and uncontrolled inflammatory response. Extracellular kinase ERK is also considered to play an important role in NF- $\kappa \mathrm{B}$ signaling pathway (Jiang et al. 2004; Ang et al. 2011). Jiang et al. (2004) believed that ERK can regulate the activation of NF- $\kappa B$ by IL- $1 \beta$ and TNF- $\alpha$, thus NF- $\kappa B$ can maintain its activation status for a longer time. Therefore, we concluded that LPS may cause ERK phosphorylation and NF- $\kappa \mathrm{B}$ activation through TLR4, and then lead to the release of inflammatory factors and the increase of apoptosis, while inflammatory factors can directly promote apoptosis and further activate NF- $\kappa B$. Apelin-13 may inhibit the inflammatory response and apoptosis through TLR4/ERK/NF- $\kappa \mathrm{B}$ signaling pathway, thus play a protective role in LPS-induced cardiac dysfunction.

Our study also found that LPS induced the increase of autophagy, while the injection of apelin-13 further increased the level of autophagy, which could be antagonized by F13A. Some studies have shown that autophagy has protective effect on sepsis related cardiac dysfunction (Hsieh et al. 2011; Sun et al. 2018). Autophagy can interact with inflammation and apoptosis through NF- $\mathrm{BB}$ (Sun et al. 2019). Therefore, we believe that apelin-13 may also protect cardiac function by improving autophagy levels.

\section{Role of F13A}

F13A is the isomer of apelin-13. Lee et al. (2005) showed that F13A can antagonize the antihypertensive effect of apelin-13, 
A

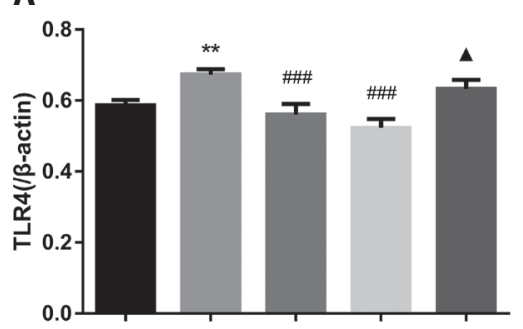

C

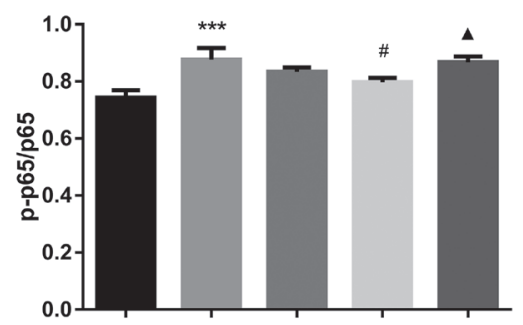

E

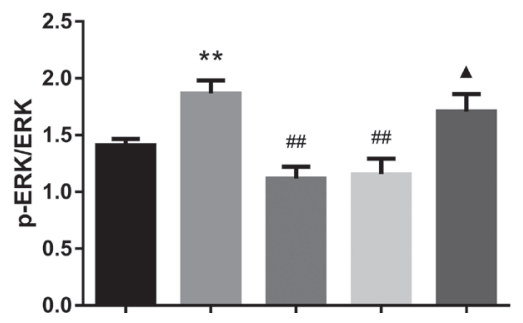

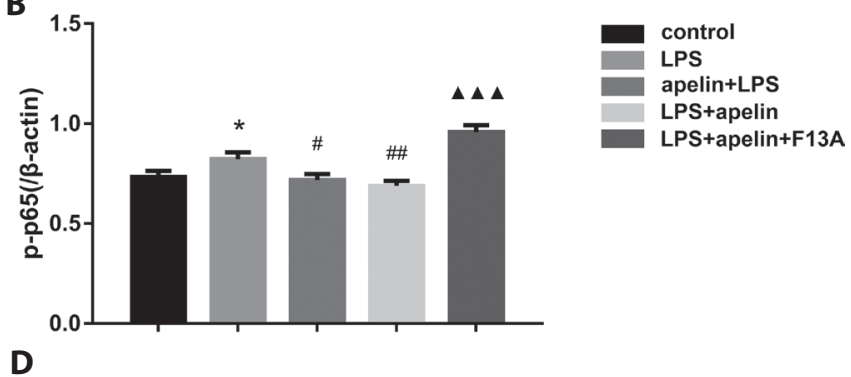

D
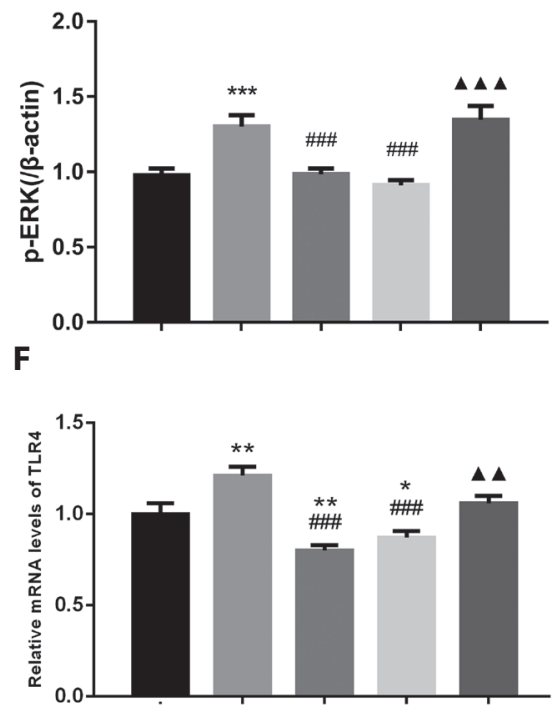

Figure 6. The effects of apelin-13 and F13A intervention on the expression of TLR4/ $\beta$-actin (A), P-p65/ $\beta$-actin (B), P-p65/p65 (C) p-ERK/ $\beta$-actin (D) and p-ERK/ERK (E) measured by Western blot, and the mRNA levels of TLR4 (F) in myocardium of LPS challenged mice. ${ }^{*} p<0.05,{ }^{* *} p<0.01$, ${ }^{* * *} p<0.001$ vs. control group; ${ }^{\#} p<$ $0.05,{ }^{\# \#} p<0.01,{ }^{\# \# \#} p<0.001$ vs. LPS group; ${ }^{\mathbf{\Delta}} p<0.05, \boldsymbol{\Delta} \mathbf{\Delta} p<0.001, \mathbf{\Delta} \boldsymbol{\Delta} \mathbf{\Delta} p<$ $0.001 v s$. LPS+apelin group. and could be used as a specific antagonist of apelin-13/APJ in a dose-dependent manner. In our study, we found that the administration of F13A at the same time of apelin-13 after LPS injection significantly antagonized the effect of apelin-13 on LPS intervention, suggesting that F13A has an antagonistic effect on exogenous apelin-13. However, F13A was given before LPS injection, and no further increase of inflammatory factors and aggravation of sepsis were found in mice. It showed that $\mathrm{F} 13 \mathrm{~A}$ has no obvious antagonistic effect on endogenous apelin under the condition of this study, but it is related to the dose and time of drug intervention, which needs further study.

\section{Limitation}

Due to the limitation of funds and the number of experimental animals, no survival rate study was conducted, and no related study was conducted on different intervention doses and different time nodes. However, inflammatory factors, apoptosis and other indicators will change in different time periods, which may affect the judgment of research results. The antagonistic effect of F13A on apelin-13 was dose-dependent. Different doses and administration time may affect the experimental results.

\section{Conclusions}

Endogenous apelin may have a protective effect on SIMD induced by LPS. Exogenous administration of apelin may inhibit LPS induced inflammatory response, apoptosis and increase autophagy through TLR4/ERK1/2/NF- $\kappa \mathrm{B}$ pathway.

Conflict of interest. The authors declare that they have no competing interests.

\section{References}

Ang SF, Moochhala SM, MacAry PA, Bhatia M (2011): Hydrogen sulfide and neurogenic inflammation in polymicrobial sepsis: involvement of substance P and ERK-NF-kappaB signaling. PLoS One 6, e24535

https://doi.org/10.1371/journal.pone.0024535

Ashley EA, Powers J, Chen M, Kundu R, Finsterbach T, Caffarelli A, Deng A, Eichhorn J, Mahajan R, Agrawal R, et al. (2005): The endogenous peptide apelin potently improves cardiac contractility and reduces cardiac loading in vivo. Cardiovasc. Res. 65, 73-82 https://doi.org/10.1016/j.cardiores.2004.08.018

Balija TM, Lowry SF (2011): Lipopolysaccharide and sepsis-associated myocardial dysfunction. Curr. Opin. Infect. Dis. 24, 248-253 
https://doi.org/10.1097/QCO.0b013e32834536ce

Baumgarten G, Knuefermann P, Schuhmacher G, Vervolgyi V, von Rappard J, Dreiner U, Fink K, Djoufack C, Hoeft A, Grohe C, et al. (2006): Toll-like receptor $\mathbf{4}$, nitric oxide, and myocardial depression in endotoxemia. Shock 25, 43-49 https://doi.org/10.1097/01.shk.0000196498.57306.a6

Bentzer P, Russell JA, Walley KR (2015): Advances in sepsis research. Clin. Chest. Med. 36, 521-530 https://doi.org/10.1016/j.ccm.2015.05.009

Biering-Sorensen T, Solomon SD (2015): Assessing contractile function when ejection fraction is normal: A case for strain imaging. Circ. Cardiovasc. Imaging 8, e004181 https://doi.org/10.1161/CIRCIMAGING.115.004181

Chagnon F, Coquerel D, Salvail D, Marsault E, Dumaine R, AugerMessier M, Sarret P, Lesur O (2017): Apelin compared with dobutamine exerts cardioprotection and extends survival in a rat model of endotoxin-induced myocardial dysfunction. Crit. Care Med. 45, e391-e398 https://doi.org/10.1097/CCM.0000000000002097

Charo DN, Ho M, Fajardo G, Kawana M, Kundu RK, Sheikh AY, Finsterbach TP, Leeper NJ, Ernst KV, Chen MM, et al. (2009): Endogenous regulation of cardiovascular function by apelinAPJ. Am. J. Physiol. Heart Circ. Physiol. 297, H1904-1913 https://doi.org/10.1152/ajpheart.00686.2009

Coquerel D, Chagnon F, Sainsily X, Dumont L, Murza A, Cote J, Dumaine R, Sarret P, Marsault E, Salvail D, et al. (2017): ELABELA improves cardio-renal outcome in fatal experimental septic shock. Crit. Care Med. 45, e1139-e1148 https://doi.org/10.1097/CCM.0000000000002639

Dillenseger L, Langlet C, Iacobelli S, Lavaux T, Ratomponirina C, Labenne M, Astruc D, Severac F, Gouyon JB, Kuhn P (2018): Early inflammatory markers for the diagnosis of late-onset sepsis in neonates: the nosodiag study. Front. Pediatr. 6, 346 https://doi.org/10.3389/fped.2018.00346

Farkasfalvi K, Stagg MA, Coppen SR, Siedlecka U, Lee J, Soppa GK, Marczin N, Szokodi I, Yacoub MH, Terracciano CM (2007): Direct effects of apelin on cardiomyocyte contractility and electrophysiology. Biochem. Biophys. Res. Commun. 357, 889-895 https://doi.org/10.1016/j.bbrc.2007.04.017

Fleischmann C, Scherag A, Adhikari NK, Hartog CS, Tsaganos T, Schlattmann P, Angus DC, Reinhart K, International Forum of Acute Care Trialists (2016): Assessment of global incidence and mortality of hospital-treated sepsis. Current estimates and limitations. Am. J. Respir. Crit. Care Med. 193, 259-272 https://doi.org/10.1164/rccm.201504-0781OC

Flierl MA, Rittirsch D, Huber-Lang MS, Sarma JV, Ward PA (2008): Molecular events in the cardiomyopathy of sepsis. Mol. Med. 14, 327-336 https://doi.org/10.2119/2007-00130.Flierl

Fong Y, Lowry SF (1990): Tumor necrosis factor in the pathophysiology of infection and sepsis. Clin. Immunol. Immunopathol. 55, 157-170 https://doi.org/10.1016/0090-1229(90)90094-7

Fry DE (2012): Sepsis, systemic inflammatory response, and multiple organ dysfunction: the mystery continues. Am. Surg. 78, 1-8 https://doi.org/10.1177/000313481207800102
Galanth C, Hus-Citharel A, Li B, Llorens-Cortes C (2012): Apelin in the control of body fluid homeostasis and cardiovascular functions. Curr. Pharm. Des. 18, 789-798

https://doi.org/10.2174/138161212799277770

Gasparini C, Feldmann M (2012): NF-kappaB as a target for modulating inflammatory responses. Curr. Pharm. Des. 18, $5735-5745$ https://doi.org/10.2174/138161212803530763

Huang Q, Liu X, Cao C, Lei J, Han D, Chen G, Yu J, Chen L, Lv D, Li Z (2016): Apelin-13 induces autophagy in hepatoma HepG2 cells through ERK1/2 signaling pathway-dependent upregulation of Beclin1. Oncology Letters 11, 1051-1056 https://doi.org/10.3892/ol.2015.3991

Hsieh CH, Pai PY, Hsueh HW, Yuan SS, Hsieh YC (2011): Complete induction of autophagy is essential for cardioprotection in sepsis. Ann. Surg. 253, 1190-1200 https://doi.org/10.1097/SLA.0b013e318214b67e

Jardin F, Fourme T, Page B, Loubieres Y, Vieillard-Baron A, Beauchet A, Bourdarias JP (1999): Persistent preload defect in severe sepsis despite fluid loading: A longitudinal echocardiographic study in patients with septic shock. Chest 116, 1354-1359 https://doi.org/10.1378/chest.116.5.1354

Jawad I, Luksic I, Rafnsson SB (2012): Assessing available information on the burden of sepsis: global estimates of incidence, prevalence and mortality. J. Glob. Health 2, 010404 https://doi.org/10.7189/jogh.01.010404

Jiang B, Xu S, Hou X, Pimentel DR, Brecher P, Cohen RA (2004): Temporal control of NF-kappaB activation by ERK differentially regulates interleukin-1beta-induced gene expression. J. Biol. Chem. 279, 1323-1329 https://doi.org/10.1074/jbc.M307521200

Kleinz MJ, Davenport AP (2005): Emerging roles of apelin in biology and medicine. Pharmacol. Ther. 107, 198-211 https://doi.org/10.1016/j.pharmthera.2005.04.001

Kumar A, Brar R, Wang P, Dee L, Skorupa G, Khadour F, Schulz R, Parrillo JE (1999): Role of nitric oxide and cGMP in human septic serum-induced depression of cardiac myocyte contractility. Am. J. Physiol. 276, R265-276 https://doi.org/10.1152/ajpregu.1999.276.1.R265

Landesberg G, Gilon D, Meroz Y, Georgieva M, Levin PD, Goodman S, Avidan A, Beeri R, Weissman C, Jaffe AS, et al. (2012): Diastolic dysfunction and mortality in severe sepsis and septic shock. Eur. Heart J. 33, 895-903 https://doi.org/10.1093/eurheartj/ehr351

Lee DK, Saldivia VR, Nguyen T, Cheng R, George SR, O‘Dowd BF (2005): Modification of the terminal residue of apelin-13 antagonizes its hypotensive action. Endocrinology 146, 231-236 https://doi.org/10.1210/en.2004-0359

Levy RJ, Piel DA, Acton PD, Zhou R, Ferrari VA, Karp JS, Deutschman CS (2005): Evidence of myocardial hibernation in the septic heart. Crit. Care Med. 33, 2752-2756 https://doi.org/10.1097/01.CCM.0000189943.60945.77

Li HR, Liu J, Zhang SL, Luo T, Wu F, Dong JH, Guo YJ, Zhao L (2017): Corilagin ameliorates the extreme inflammatory status in sepsis through TLR4 signaling pathways. BMC Complement. Altern. Med. 17, 18 https://doi.org/10.1186/s12906-016-1533-y 
Michie HR, Manogue KR, Spriggs DR, Revhaug A, O'Dwyer S, Dinarello CA, Cerami A, Wolff SM, Wilmore DW (1988): Detection of circulating tumor necrosis factor after endotoxin administration. N. Engl. J. Med. 318, 1481-1486 https://doi.org/10.1056/NEJM198806093182301

Oeckinghaus A, Hayden MS, Ghosh S (2011): Crosstalk in NFkappaB signaling pathways. Nat. Immunol. 12, 695-708 https://doi.org/10.1038/ni.2065

Piper RD, Li FY, Myers ML, Sibbald WJ (1997): Structure-function relationships in the septic rat heart. Am. J. Respir. Crit. Care Med. 156, 1473-1482 https://doi.org/10.1164/ajrccm.156.5.96-10085

Suffredini AF, Fromm RE, Parker MM, Brenner M, Kovacs JA, Wesley RA, Parrillo JE (1989): The cardiovascular response of normal humans to the administration of endotoxin. N. Engl. J. Med. 321, 280-287 https://doi.org/10.1056/NEJM198908033210503

Sullivan JS, Kilpatrick L, Costarino AT Jr, Lee SC, Harris MC (1992): Correlation of plasma cytokine elevations with mortality rate in children with sepsis. J. Pediatr. 120, 510-515 https://doi.org/10.1016/S0022-3476(05)82476-X

Sun Y, Cai Y, Zang QS (2019): Cardiac autophagy in sepsis. Cells 8, 141 https://doi.org/10.3390/cells8020141

Sun Y, Yao X, Zhang QJ, Zhu M, Liu ZP, Ci B, Xie Y, Carlson D, Rothermel BA, Sun Y, et al. (2018): Beclin-1-dependent autophagy protects the heart during sepsis. Circulation 138, 2247-2262 https://doi.org/10.1161/CIRCULATIONAHA.117.032821

Szokodi I, Tavi P, Foldes G, Voutilainen-Myllyla S, Ilves M, Tokola H, Pikkarainen S, Piuhola J, Rysa J, Toth M, et al. (2002): Apelin, the novel endogenous ligand of the orphan receptor APJ, regulates cardiac contractility. Circ. Res. 91, 434-440
https://doi.org/10.1161/01.RES.0000033522.37861.69

Takeuchi O, Akira S (2010): Pattern recognition receptors and inflammation. Cell 140, 805-820 https://doi.org/10.1016/j.cell.2010.01.022

Tatemoto K, Hosoya M, Habata Y, Fujii R, Kakegawa T, Zou MX, Kawamata Y, Fukusumi S, Hinuma S, Kitada C, et al. (1998): Isolation and characterization of a novel endogenous peptide ligand for the human APJ receptor. Biochem. Biophys. Res. Commun. 251, 471-476 https://doi.org/10.1006/bbrc.1998.9489

Vaez H, Rameshrad M, Najafi M, Barar J, Barzegari A, Garjani A (2016): Cardioprotective effect of metformin in lipopolysaccharide-induced sepsis via suppression of toll-like receptor 4 (TLR4) in heart. Eur. J. Pharmacol. 772, 115-123 https://doi.org/10.1016/j.ejphar.2015.12.030

Xu W, Li T, Gao L, Zheng J, Yan J, Zhang J, Shao A (2019): Apelin-13/APJ system attenuates early brain injury via suppression of endoplasmic reticulum stress-associated TXNIP/NLRP3 inflammasome activation and oxidative stress in a AMPKdependent manner after subarachnoid hemorrhage in rats. J. Neuroinflammation 16, 247 https://doi.org/10.1186/s12974-019-1620-3

Yang XX, Huang TH, Liu HM, Huang Y (2017): NF- $\kappa B$ participates in the regulation of apoptosis. Journal of Shanghai Jiao Tong University (Medical Science) 37, 1446-1452

Zhang WB, Zhang HY, Zhang Q, Jiao FZ, Zhang H, Wang LW, Gong ZJ (2017): Glutamine ameliorates lipopolysaccharide-induced cardiac dysfunction by regulating the toll-like receptor 4/ mitogen-activated protein kinase/nuclear factor-kB signaling pathway. Exp. Ther. Med. 14, 5825-5832 https://doi.org/10.3892/etm.2017.5324

Received: November 20, 2020

Final version accepted: March 3, 2021 